\title{
Desempenho de clones de seringueira da série IAC 300 selecionados para a região noroeste do Estado de São Paulo(1)
}

\author{
Paulo de Souza Gonçalves ${ }^{(2)}$, Nelson Bortoletto(3), Edson Luiz Furtado(4), \\ Rosana Sambugaro( ${ }^{(5)}$ e Ondino Cleante Bataglia(6)
}

Resumo -É apresentado o desempenho de 19 novos clones de seringueira [Hevea brasiliensis (Willd. ex Adr. de Juss.) Muell. Arg.], resultantes de hibridações conduzidas no Instituto Agronômico e avaliados em experimento de pequena escala, tendo o clone RRIM 600 como testemunha. O experimento em campo obedeceu ao delineamento em blocos ao acaso, com três repetições. Com relação à produção de borracha seca, o clone IAC 40 apresentou a maior média (62,22 g/árvore/sangria) nos três anos de avaliação, seguido pelo IAC 301 (57,67 g/árvore/sangria) e pelo IAC 300 (50,61 g/árvore/sangria), com produções 154\%, 138\% e 123\% superiores em relação ao RRIM 600 (41,04 g/árvore/sangria). Todos os clones selecionados foram vigorosos, com perímetro do caule na abertura do painel variando de $37,81 \mathrm{~cm}$ (IAC 317) a 50,90 cm (IAC 315). A porcentagem de plantas aptas a sangria variou de $20,0 \%$ (IAC 317) a 100\% (IAC 315). Todos os clones apresentaram baixas incidências de quebra pelo vento e de secamento do painel. Não foi detectada nenhuma doença foliar em caráter epidêmico. Dos clones estudados, 15 apresentaram alta resistência à antracnose do painel, e foram superiores ao RRIM 600; os outros cinco apresentaram resistência moderada semelhante ao RRIM 600.

Termos para indexação: Hevea brasiliensis, características agronômicas, borracha, rendimento

\section{Performance of Hevea clones from IAC 300 series selected in the northwestern region of São Paulo State, Brazil}

\begin{abstract}
The present paper shows the performance of 19 rubber tree [Hevea brasiliensis (Willd. ex Adr. de Juss.) Muell. Arg.] originated from Hevea breeding programme conducted at the Instituto Agronômico de Campinas (IAC) and evaluated in a small scale trial in the northwestern of São Paulo State, Brazil. The old popular clone RRIM 600 of Malaysian origin was used as control. The trial was laid out in a randomized block design with three replications. Concerned to yielding the clone IAC 40 recorded highest yield ( $62.22 \mathrm{~g} /$ tree/tap) over three years of tapping which was followed by IAC 301 (57.67 g/tree/tap) and IAC 300 (50.61 g/tree/tap) yielding 154\%, 138\% and $123 \%$ superior to the control clone and RRIM 600 whereas recorded 41.04 tree/tap. All selected clones were of vigorous growing, with the girth at opening ranging from $37.81 \mathrm{~cm}$ for IAC 317 to $50.90 \mathrm{~cm}$ for IAC 315 and tappability ranging from $20.0 \%$ for IAC 317 to $100 \%$ for IAC 315 . All the clones experienced low to very low incidence of wind damage. All selected clones showed low to very low incidence of dry trees. Fifteen clones showed high resistance and five presented moderate resistance to anthrachnose panel canker disease similarly to RRIM 600.
\end{abstract}

Index terms: rubber tree, Hevea brasiliensis, agronomic characters, rubber, yields

(1) Aceito para publicação em 12 de julho de 2000.

Pesquisa executada com recursos parciais da FAPESP.

(2) Embrapa/Instituto Agronômico (IAC), Centro de Café e Plantas Tropicais (CCPT), Caixa Postal 28, CEP 13001-970 Campinas, SP. Bolsista do CNPq. E-mail: paulog@cec.iac.br

(3)IAC, Núcleo de Agronomia do Noroeste, Caixa Postal 401, CEP15500-000 Votuporanga, SP.E-mail:nan.iac@votuporanga.com.br

(4)UNESP, Faculdade de Ciências Agronômicas, Caixa Postal 237, CEP 18603-970 Botucatu, SP. E-mail: elfurtado@fca.unesp.br

(5) IAC, CCPT. Bolsista da FAPESP.

E-mail: rosanasambugaro@lycos.com

(6)IAC, Centro de Solos e Recursos Ambientais, Campinas, SP E-mail: ondino@cec.iac.br

\section{Introdução}

A seringueira pertence ao gênero Hevea, da família Euphorbiaceae, e tem a Hevea brasiliensis (Willd. ex Adr. de Juss.) Muell. Arg. como a espécie mais importante do gênero. Na Ásia, ela é plantada como a principal fonte de borracha natural, sendo que em 1998 mais de $72 \%$ da produção mundial foi originária da Tailândia, Indonésia e Malásia. A área total estimada de seringueira plantada no globo é superior 
a 9 milhões de hectares, tradicionalmente cultivada na região equatorial, situada entre $10^{\circ}$ Norte e $10^{\circ}$ Sul do Equador.

A despeito de ser o berço das espécies desse gênero, o Brasil contribuiu em 1998, com apenas 1\% da produção mundial de 6.680 mil toneladas para um consumo em torno de $3 \%$ de um total de 6.610 mil toneladas da demanda mundial (International Rubber Study Group, 1999).

No Brasil, a história da produção da borracha vegetal mostra que o País desfrutou da condição de principal produtor e exportador mundial no final do século passado e início do atual, passando a ser importador desta matéria-prima a partir do início dos anos cinqüenta. Ressalta-se, também, que em 1998, a produção brasileira foi estimada em 70 mil toneladas, para um consumo de 195 mil toneladas, e que, menos de 5\% da borracha produzida no País foi proveniente de seringais nativos.

Para um país que possui, em relação aos demais produtores, área apta, incomparavelmente maior, para o plantio de seringueira, o déficit de produção significa, no mínimo, descaso para um produto estratégico de tão alto valor econômico-social. Particularizando as áreas de escape, só o Estado de São Paulo possui 14 milhões de hectares aptos à heveicultura, dos quais apenas desse total cerca de 44,6 mil hectares estavam ocupados com seringueiras em 1997 (Vazquez Cortez, 1999), conferindo ao Estado a condição de primeiro produtor de borracha natural do Brasil, com uma produção estimada para 1998 de 35 mil toneladas, $50 \%$ da produção nacional.

Há cerca de 120 anos, quando teve início sua domesticação na Ásia (Gonçalves et al., 1983), a seringueira foi considerada mais uma espécie selvagem da Amazônia. A partir desse período, o melhoramento genético tem contribuído para seu desenvolvimento, elevando o nível da produção de $400 \mathrm{~kg}$ para $2.500 \mathrm{~kg} / \mathrm{ha} /$ ano de borracha seca.

Hibridações seletivas entre clones superiores de seringueira, seguidas da multiplicação vegetativa do ortete selecionado e posterior avaliação, têm sido a base do melhoramento genético no Estado de São Paulo. Devido ao longo ciclo do melhoramento, aproximadamente, de 30 anos (Gonçalves et al., 1988), esforços no sentido de sua redução incluem o desenvolvimento de métodos de seleção precoce, redução dos ciclos de seleção, e exploração do potencial dos parentais.

Entretanto, à medida que novas fronteiras são abertas com essa cultura exótica, aumentam as preocupações relativas ao limitado número de clones plantados, sujeitos a vulnerabilidade, necessitando da ampliação do programa de melhoramento genético quanto às futuras recomendações de clones para o plantio.

O objetivo deste trabalho foi apresentar os resultados de três anos de produção de borracha da primeira seleção de clones da nova série IAC 300, bem como os de outros caracteres importantes da segunda seleção de clones em experimentos de avaliação de pequena escala.

\section{Material e Métodos}

Dezenove clones obtidos de polinizações controladas (Tabela 1) foram instalados em um experimento de avaliação de clones no Núcleo de Agronomia do Noroeste (NAN) do Instituto Agronômico (IAC), estabelecido no Município de Votuporanga, SP, a $20^{\circ} 20^{\prime}$ S de latitude, $49^{\circ} 58^{\prime} \mathrm{W}$ de longitude e $510 \mathrm{~m}$ de altitude, em solo Podzólico Vermelho-Escuro, latossólico eutrófico a moderado, textura arenosa média.

Predomina, na região, o clima Aw (Köppen), com estação seca definida, temperatura média anual de $22,2^{\circ} \mathrm{C}$, amplitude média de $18,0^{\circ} \mathrm{C}$ a $24,1^{\circ} \mathrm{C}$, umidade relativa média anual em torno de $70,0 \%$, com extremos de $77,1 \%$ em fevereiro e $59,0 \%$ em agosto. A pluviosidade média anual é em torno de $1.370 \mathrm{~mm}$, com regime tropical de $74,0 \%$ de outubro a março e $26 \%$ de abril a setembro

Adotou-se o delineamento em blocos ao acaso, com três repetições, sendo cada parcela representada por seis árvores plantadas no espaçamento de 7,0 m entre linhas e 3,0 m entre plantas

Durante o período de crescimento dos clones, mensurações anuais de perímetro do caule foram feitas para determinação do vigor a $0,50 \mathrm{~m}$ acima do calo de enxertia, no primeiro ano, e a $1,20 \mathrm{~m}$ a partir do segundo ano durante dez anos consecutivos. Foi utilizado na análise conjunta do incremento do caule obtido pela diferença das avaliações posteriores com as anteriores, e conduzidas análises combinadas utilizando-se o delineamento de blocos ao acaso, com parcelas subdivididas no tempo, uma vez que foram efetuadas medidas sucessivas numa mesma parcela, num certo período de tempo.

Dados de produção foram registrados a partir do sétimo ano de idade em relação a todas as árvores da parcela, 
que apresentaram perímetro do caule superior a $45 \mathrm{~cm}$ O registro da produção foi efetuado por meio do látex coagulado nas tigelas, coletado ao acaso duas vezes ao mês, secado em condições normais de sombra e ventilação ao longo do período de avaliação, preso a cada árvore. O total anual de borracha por árvore foi então dividido pelo número de "coágulos", sendo o resultado expresso em gramas/árvore/sangria. A média geral do total árvores/clone foi expressa em gramas/árvore/clone/ano. A partir desses dados, estimou-se a produção por clone/hectare/ano, seguindo recomendações da Embrapa (1989), e adotou-se o total de 140 sangrias por ano: no primeiro ano, foram utilizadas, para cálculo, 240 plantas (60\% do stand); no segundo ano, 340 plantas ( $85 \%$ do stand), e no terceiro ano, 380 plantas (95\% do stand).

Para cada avaliação anual da produção foram utilizados diferentes sistemas de sangria. Foram realizadas análises de variância individual com média de parcela de cada avaliação. No primeiro ano, o sistema utilizado foi o $1 / 2 \mathrm{~S} \mathrm{~d} / 25 \mathrm{~d} / 7.11 \mathrm{~m} / \mathrm{y}$ - sangria em meia espiral $(1 / 2 \mathrm{~S})$, realizada em intervalos de dois dias $(\mathrm{d} / 2)$; a atividade de sangria foi realizada em 5 dias na semana $(5 \mathrm{~d} / 7)$, durante 11 meses por ano $(11 \mathrm{~m} / \mathrm{y})$; no segundo ano, o sistema utilizado foi $1 / 2 \mathrm{~S} \mathrm{~d} / 35 \mathrm{~d} / 7$. $11 \mathrm{~m} / \mathrm{y}$. ET $2,5 \% \mathrm{~Pa} .10 / \mathrm{y}$ - sangria em meia espiral $(1 / 2 \mathrm{~S})$, realizada em intervalo de três dias (d/3); a atividade foi realizada em cinco dias da semana $(5 \mathrm{~d} / 7)$, durante 11 meses do ano $(11 \mathrm{~m} / \mathrm{y})$ com estimulação feita com ethefon a 2,5\% do ingrediente ativo (ET 2,5\%), aplicado por pincelamento no painel de sangria $(\mathrm{Pa}) \mathrm{dez}$ vezes por ano $(10 / y)$, e, finalmente, no terceiro ano, foi utilizado o sistema $1 / 2 \mathrm{~S} \mathrm{~d} / 45 \mathrm{~d} / 7$. $11 \mathrm{~m} / \mathrm{y}$. ET $5,0 \% \mathrm{~Pa} 8 / \mathrm{y}-$ sangria em meia espiral $(1 / 2 \mathrm{~S})$, realizada em intervalos de quatro dias $(\mathrm{d} / 4)$; a atividade foi realizada nos cinco dias da semana $(5 \mathrm{~d} / 7)$ durante 11 meses do ano $(11 \mathrm{~m} / \mathrm{y})$, com estimulação feita com ethefon a $5,0 \%$ aplicado por pincelamento no painel de sangria $(\mathrm{Pa})$, oito vezes por ano (8/y). Para a estimativa da produção por hectare/ano de cada clone e sistema de sangria, adotaram-se 140 sangrias no sistema $\mathrm{d} / 2,100$ sangrias no sistema $\mathrm{d} / 3$, e 72 sangrias no sistema d/4 de acordo com Virgens Filho et al. (1999).

Amostras de casca para determinação de espessura da casca virgem foram tomadas a $1,00 \mathrm{~m}$ de altura do calo de cada árvore, com auxílio de um vazador, e, em seguida, medidas com paquímetro em laboratório, e o número de anéis de vasos laticíferos obtidos através das secções longitudinais radiais da casca.

Durante a execução do experimento, foram empregadas todas as práticas de manejo convencionais ao cultivo da seringueira (São Paulo, 1999), exceto o controle fitossanitário.

Tabela 1. Paternais dos clones referentes à primeira seleção da série IAC realizada no Núcleo de Agronomia do Noroeste do Instituto Agronômico (IAC), Votuporanga, $\mathrm{SP}^{(1)}$.

\begin{tabular}{|c|c|}
\hline Clones & Parentais \\
\hline IAC 40 & RRIM 608 (AVROS 33 x Tjir 1) x AVROS 1279 (AVROS 156 x AVROS 374) \\
\hline IAC 56 & RRIM 608 (AVROS 33 x Tjir 1) x Fx 3810 (F 4542 x AVROS 363) \\
\hline IAC 300 & RRIM 605 (Tjir 1 x PB 49) x AVROS 363 \\
\hline IAC 301 & RRIM 501 (Pil 49 x Lun N) x AVROS 1518 \\
\hline IAC 302 & RRIM 501 (Pil A 49 x Lun N) x AVROS 353 \\
\hline IAC 303 & RRIM 511 (Pil B 84 x Pil A 44) x AVROS 1218 (AVROS 214 x AVROS 216) \\
\hline IAC 306 & AVROS 49 x RRIM 509 (Pil A 44 x Pil B 246) \\
\hline IAC 307 & AVROS 1328 (AVROS 214 x AVROS 317) x PR 107 \\
\hline IAC 308 & AVROS 49 x PR 107 \\
\hline IAC 309 & RRIM 626 (Tjir 1 x RRIM 600) x Fx 25 (F 351 x AVROS 49) \\
\hline IAC 310 & AVROS 1328 (AVROS 214 x AVROS 317) x PB 86 \\
\hline IAC 311 & AVROS 509 (Pil A 44 x Lun N) x Fx 25 (F 351 x AVROS 49) \\
\hline IAC 312 & RRIM 600 (Tjir 1 x PB 86) x Fx 25 (F 351 x AVROS 49) \\
\hline IAC 313 & RRIM 626 (Tjir 1 x PB 49) x Fx 25 (F 351 x AVROS 49) \\
\hline IAC 314 & AVROS 1328 (AVROS 214 x AVROS 317) x Fx 25 (F 351 x AVROS 49) \\
\hline IAC 315 & AVROS 1328 (AVROS 214 x AVROS 317) x AVROS 49 \\
\hline IAC 316 & AVROS 1328 (AVROS 214 x AVROS 317) x RRIM 608 (Tjir 33 x Tjir 1) \\
\hline IAC 317 & AVROS 1328 (AVROS 214 x AVROS 317) x RRIM 526 (Pil B 58 x Pil B 65) \\
\hline IAC 318 & RRIM 600 (Tjir 1 x PB 86) Fx 3899 (F 4542 x AVROS 363) \\
\hline RRIM 600 & Tjir x PB 86 \\
\hline
\end{tabular}

(1)IAC: Instituto Agronômico de Campinas; RRIM: Rubber Research Institute of Malaysia; PB: Prang Besar; Tjir: Tjirandji; AVROS: Algemeine Vereinigung Rubber Planters Oostkust Sumatra; F: Ford; Pil: Pilmoor; Lun: Lunderston; Fx: Cruzamento Ford; PR: Proefstation voor Rubber. 
Na avaliação dos clones, realizada a partir da abertura do painel, a incidência natural da antracnose do painel causada pelo fungo Colletotrichum gloeosporioides (Penz.) Sacc., adotou-se, em todas as plantas, o sistema de grau de severidade, conforme a intensidade de sintomas da doença nos painéis, para isso, utilizou-se a média dos anos de avaliação e uma escala de notas graduais de 0 a 4 (AR - altamente resistente; $\mathrm{R}$ - resistente; $\mathrm{MR}$ - moderadamente resistente; $\mathrm{S}$ - suscetível; AS - altamente suscetível), e os dados foram transformados em índice de infecção, proposto por McKeney, citado por Bajungu (1977) com:

Índice de infecção $=\frac{\sum\left(\text { grau da escala } \mathrm{x} \mathrm{n}^{\circ} \text { de plantas da escala }\right)}{\mathrm{N}^{\circ} \text { de plantas avaliadas } \mathrm{x} \text { grau máximo }}$.

As observações obtidas em 20 clones de seringueira incluíram crescimento do caule e produção de borracha seca em três anos de sangria. Esses clones, resultado de uma primeira seleção efetuada em um grupo de 75 clones, foram conduzidos com base em suas produções de borracha e vigor. Para efeito de comparação, utilizou-se como testemunha o clone comercial RRIM 600. Os clones foram observados quanto ao hábito de troca de folhas: denominados de regular, quando com reenfolhamento uniforme; irregular, quando estavam presentes, nas plantas, folhas em vários estádios fenológicos, inclusive folhas velhas, conforme método descrito por Furtado (1990).

\section{Resultados e Discussão}

Houve diferenças altamente significativas $(\mathrm{p}<0,01)$ entre clones, o que indica a existência de variabilidade nos três anos de produção (Tabela 2). Não foi considerada a análise agrupada, uma vez que em cada ano foi utilizado diferente sistema de sangria, optando-se, dessa forma, pela análise de variância individual.

Sob o sistema $1 / 2 \mathrm{~S} \mathrm{~d} / 25 \mathrm{~d} / 7$, os clones IAC 40 , IAC 300, IAC 302 e IAC 301 foram os que mais produziram no primeiro ano de sangria, com $1.259 \mathrm{~kg}$, $780 \mathrm{~kg}, 774 \mathrm{~kg}$ e $732 \mathrm{~kg}$ de borracha seca por hectare/ano respectivamente, todos superiores ao RRIM 600, que apresentou média de 614 kg (Tabela 3 ).

O clone IAC 40, considerado o melhor, apresentou, no primeiro ano de sangria, produção duas vezes superior ao de RRIM 600, cuja produtividade de $614 \mathrm{~kg} / \mathrm{ha} /$ ano mostrou-se compatível com a obtida na Malásia (668 kg/ha/ano) no primeiro ano de produção (Ong et al., 1981).
No segundo ano, sob o sistema $1 / 2 \mathrm{~S} d / 3$ $5 \mathrm{~d} / 7$. ET $2,5 \%$, houve um aumento na produção de todos os clones, graças à estimulação. O clone IAC 40 foi o que mais produziu, com $2.601 \mathrm{~kg} / \mathrm{ha} / \mathrm{ano}$, seguido pelos IAC 302, IAC 300 e IAC 301 com produções de $2.013 \mathrm{~kg}, 1.970 \mathrm{~kg}$ e $1.952 \mathrm{~kg}$ de borracha seca/ha/ano, respectivamente (Tabela 3 ).

No terceiro ano, sob sistema $1 / 2 \mathrm{~S} \mathrm{~d} / 4$ $5 \mathrm{~d} / 7$. ET 5,0\%, o clone IAC 301 foi o mais produtivo, com $2.567 \mathrm{~kg} / \mathrm{ha} / \mathrm{ano}$, seguido pelos IAC 40 , IAC 300 e IAC 302 com $1.989 \mathrm{~kg}, 1.934 \mathrm{~kg}$ e $1.767 \mathrm{~kg}$ de borracha seca por hectare/ano, respectivamente. Houve queda na produção em alguns clones, talvez relacionada com a alta porcentagem de estimulante associado ao sistema adotado. Segundo Jonge (1955), a resposta à concentração de estimulação em relação à produção varia consideravelmente entre clones, variação, esta, também constatada posteriormente por Levandowsky (1961), Ho et al. (1973a) e Abraham et al. (1976).

A média dos três anos de produção ( $\mathrm{kg} / \mathrm{ha} / \mathrm{ano})$ em relação à testemunha (RRIM 600), nos clones que se destacaram, variou de $108 \%$ a $154 \%$. O clone mais produtivo foi o IAC 40, com $1.950 \mathrm{~kg} / \mathrm{ha} / \mathrm{ano}$, ou seja, $54 \%$ superior ao RRIM 600, cuja média de produção foi $1.268 \mathrm{~kg} / \mathrm{ha} / \mathrm{ano}$. O clone seguinte foi o IAC 301 , com $1.750 \mathrm{~kg} / \mathrm{ha} / \mathrm{ano}$, seguido pelos IAC 300 , IAC 302 e IAC 56 com $1.561 \mathrm{~kg}, 1.518 \mathrm{~kg}$ e $1.366 \mathrm{~kg} / \mathrm{ha} /$ ano ou seja $38 \%, 23 \%, 20 \%$ e $8 \%$ superiores ao RRIM 600, respectivamente (Tabela 3 ).

Houve diferenças significativas $(p<0,05)$ entre clones, o que indica a existência de variabilidade do incremento do caule (Tabela 4). A presença de interação entre clones e anos $(\mathrm{p}<0,01)$ revela que os clones apresentaram diferentes incrementos nos diferentes anos.

Os coeficientes de variação experimental dentro de clones e de anos variaram de 7,05\% a 30,93\% em relação ao incremento anual do caule.

Quanto às médias do perímetro anual do caule no sétimo ano (abertura do painel), correspondente a cada clone, os clones que mais se destacaram foram o IAC 315 , IAC 312 e IAC 309, com valores de $50,90 \mathrm{~cm}, 50,50 \mathrm{~cm}$ e $49,16 \mathrm{~cm}$, respectivamente (Tabela 5). Seu rápido crescimento significa redução do período de imaturidade tornando esses clones aptos à abertura do painel aos seis anos de idade. Por 
outro lado, os clones com menores desenvolvimentos do caule foram IAC 310, RRIM 600 (testemunha) e IAC 317 , com médias de $41,10 \mathrm{~cm}, 39,28 \mathrm{~cm}$, $37,81 \mathrm{~cm}$, respectivamente.

No período de imaturidade as maiores médias anuais de crescimento do caule foram apresentadas pelos clones IAC 312 e IAC 315 ambos com 7,27 cm, seguidos pelos clones IAC 40, IAC 56 e IAC 311 com médias de $7,26 \mathrm{~cm}, 7,21 \mathrm{~cm}$ e $7,00 \mathrm{~cm}$, respectivamente (Tabela 6). No período adulto, ou seja, período de sangria, considerando que o crescimento dos clones nessa fase é muito importante para a manutenção da constância de produção, o clone IAC 310, seguido pelos clones IAC 318, IAC 303 e

Tabela 2. Valores dos quadrados médios, coeficientes de variação (CV) e média geral da produção, em gramas de borracha seca por sangria por árvore, referentes à análise de variância dos 20 clones de seringueira do experimento de avaliação em pequena escala, instalado no Núcleo de Agronomia do Noroeste do Instituto Agronômico (IAC), Votuporanga, SP.

\begin{tabular}{lcccc}
\hline Fonte de variação & GL & \multicolumn{3}{c}{ Produção $(\mathrm{g})$} \\
\cline { 3 - 5 } & & Ano $1^{(1)}$ & Ano $2^{(2)}$ & Ano $^{(3)}$ \\
\hline Blocos & 2 & 6,2096 & 39,1006 & 29,7111 \\
Clones & 19 & $75,0422^{* *}$ & $488,1068^{* *}$ & $1195,9586^{* *}$ \\
Resíduo & 38 & 20,3499 & 69,7697 & 52,4089 \\
\hline CV $(\%)$ & & 32,961 & 21,525 & 15,146 \\
Média geral & 13,69 & 38,80 & 47,80 \\
\hline
\end{tabular}

${ }^{(1)}$ Sistema de sangria $1 / 2 \mathrm{~S} \mathrm{~d} / 25 \mathrm{~d} / 7 .{ }^{(2)}$ Sistema de sangria $1 / 2 \mathrm{~S} \mathrm{~d} / 3$ 5d/7. 11m/y. ET 2,5\% Pa.10/y. ${ }^{(3)}$ Sistema de sangria $1 / 2 \mathrm{~S}$ d/4 5d/7. 11m/y. ET 5,0\% Pa. 8/y. ** Significativo a $1 \%$ de probabilidade

Tabela 3. Estimativas de produção de borracha seca e porcentagem de ganho em relação à testemunha (RRIM 600) referentes aos três anos de sangria de 20 clones selecionados no experimento de avaliação em pequena escala, na Estação Experimental de Votuporanga, SP.

\begin{tabular}{|c|c|c|c|c|c|c|c|c|c|}
\hline \multirow[t]{2}{*}{ Clone } & \multicolumn{2}{|c|}{ Ano $1^{(1)}$} & \multicolumn{2}{|c|}{ Ano $2^{(2)}$} & \multicolumn{2}{|c|}{ Ano $3^{(3)}$} & \multicolumn{2}{|c|}{ Média } & \multirow{2}{*}{$\begin{array}{l}\text { Porcentagem } \\
\text { (testemunha) }\end{array}$} \\
\hline & $\mathrm{g} / \mathrm{s} / \mathrm{a}^{(4)}$ & $\mathrm{kg} / \mathrm{ha} / \mathrm{ano}^{(5)}$ & $\mathrm{g} / \mathrm{s} / \mathrm{a}$ & $\mathrm{kg} / \mathrm{ha} / \mathrm{ano}$ & $\mathrm{g} / \mathrm{s} / \mathrm{a}$ & kg/ha/ano & $\mathrm{g} / \mathrm{s} / \mathrm{a}$ & $\mathrm{kg} / \mathrm{ha} / \mathrm{ano}$ & \\
\hline IAC 40 & 37,46 & 1.259 & 76,50 & 2.601 & 72,69 & 1.989 & 62,22 & 1.950 & 154 \\
\hline IAC 56 & 15,50 & 521 & 46,69 & 1.587 & 72,72 & 1.990 & 44,97 & 1.366 & 108 \\
\hline IAC 300 & 23,22 & 780 & 57,94 & 1.970 & 70,70 & 1.934 & 50,61 & 1.561 & 123 \\
\hline IAC 301 & 21,78 & 732 & 57,42 & 1.952 & 93,82 & 2.567 & 57,67 & 1.750 & 138 \\
\hline IAC 302 & 23,05 & 774 & 59,20 & 2.013 & 64,60 & 1.767 & 48,96 & 1.518 & 120 \\
\hline IAC 303 & 15,36 & 516 & 41,43 & 1.409 & 48,67 & 1.332 & 35,15 & 1.086 & 86 \\
\hline IAC 306 & 14,78 & 496 & 33,11 & 1.126 & 36,85 & 1.008 & 28,25 & 877 & 69 \\
\hline IAC 307 & 14,78 & 497 & 36,01 & 1.224 & 47,07 & 1.288 & 32,61 & 1.003 & 79 \\
\hline IAC 308 & 13,95 & 469 & 37,36 & 1.270 & 38,27 & 1.047 & 29,87 & 929 & 73 \\
\hline IAC 309 & 12,90 & 433 & 33,48 & 1.138 & 43,38 & 1.187 & 29,93 & 920 & 73 \\
\hline IAC 310 & 12,48 & 419 & 32,30 & 1.098 & 39,30 & 1.075 & 28,03 & 864 & 68 \\
\hline IAC 311 & 11,73 & 394 & 26,30 & 894 & 29,89 & 818 & 22,63 & 702 & 55 \\
\hline IAC 312 & 10,65 & 359 & 30,73 & 1.045 & 24,49 & 670 & 21,96 & 691 & 54 \\
\hline IAC 313 & 10,50 & 353 & 33,35 & 1.134 & 53,08 & 1.452 & 32,32 & 980 & 77 \\
\hline IAC 314 & 10,15 & 341 & 28,10 & 955 & 36,38 & 995 & 24,88 & 764 & 60 \\
\hline IAC 315 & 9,18 & 309 & 28,59 & 972 & 30,38 & 831 & 22,72 & 704 & 56 \\
\hline IAC 316 & 11,94 & 401 & 23,43 & 797 & 29,16 & 798 & 21,51 & 665 & 52 \\
\hline IAC 317 & 10,56 & 355 & 33,63 & 1.143 & 15,08 & 413 & 19,76 & 637 & 50 \\
\hline IAC 318 & 8,29 & 279 & 26,49 & 901 & 45,97 & 1.258 & 26,92 & 813 & 64 \\
\hline RRIM $600^{(6)}$ & 18,26 & 614 & 48,38 & 1.645 & 56,48 & 1.545 & 41,04 & 1.268 & 100 \\
\hline
\end{tabular}

(1) Sistema de sangria $1 / 2 \mathrm{~S} \mathrm{~d} / 25 \mathrm{~d} / 7$. (2) Sistema de sangria $1 / 2 \mathrm{~S} \mathrm{~d} / 3$ 5d/7. 11m/y. ET 2,5\% Pa.10/y. ${ }^{(3)}$ Sistema de sangria $1 / 2 \mathrm{~S} \mathrm{~d} / 4$ 5d/7. 11m/y. ET 5,0\% Pa. 8/y.

${ }^{(4)}$ Gramas de borracha seca por sangria por árvore. ${ }^{(5)} \mathrm{kg} / \mathrm{ha} / \mathrm{ano}=$ peso em gramas x no de árvores/hectare x no de sangrias/ano. ${ }^{(6)} \mathrm{Testemunha}$. 
IAC 311, apresentou a maior média de crescimento anual, com 7,33 cm, $6,61 \mathrm{~cm}, 5,60 \mathrm{~cm}$ e $5,43 \mathrm{~cm}$, respectivamente.

A média do perímetro do caule das plantas aptas à abertura dos painéis dos clones em estudo está relacionada com o porcentual de plantas aptas à sangria cujo porcentual variou de $20 \%$ (IAC 317 ) a $100 \%$ (IAC 315) (Tabela 7). Outros clones com alta porcentagem de plantas aptas à sangria foram o IAC 303, IAC 316 e IAC 312 com 95\%, 93\%, 92\%, respectivamente. Mesmo tendo em conta a variabilidade entre clones, onde as médias do perímetro variaram de $37,81 \mathrm{~cm}$ a $50,90 \mathrm{~cm}$ na abertura do painel, a média geral de $45,32 \mathrm{~cm}$ traduz um bom desenvolvimento das árvores.

As espessuras de casca virgem de todos os clones foram aceitáveis, sendo o valor mais baixo de $5,70 \mathrm{~mm}$. Os clones IAC 315 e IAC 56 apresentaram casca espessa com 7,50 mm e 7,21 mm, respectivamente, enquanto o IAC 302 e o IAC 40 apresentaram casca mais fina. Segundo Gonçalves et al. (1999), a importância dessa variável consiste em influenciar indiretamente na produtividade do clone, por proporcionar maior facilidade no procedimento da sangria.

Tabela 4. Valores dos quadrados médios da análise de variância, coeficientes de variação $(\mathrm{CV})$ e média geral do incremento do caule, em centímetros, referente ao período de 10 anos de avaliação de 20 clones do experimento de avaliação em pequena escala, instalado no Núcleo de Agronomia do Noroeste do Instituto Agronômico (IAC), Votuporanga, SP.

\begin{tabular}{lrc}
\hline Fonte de variação & GL & Incremento do caule \\
\hline Blocos & 2 & $2,3072^{\text {ns }}$ \\
Clones & 19 & $3,9474^{*}$ \\
Resíduo (clones) & 38 & 1,7999 \\
Anos & 9 & $204,6553^{* *}$ \\
Clones x Anos & 171 & $5,7702^{* *}$ \\
Anos x Blocos & 18 & $4,3068^{\text {ns }}$ \\
Resíduo (anos) & 342 & 3,4687 \\
\hline CV (\%) (clones) & & 7,05 \\
CV (\%) (anos) & & 30,93 \\
Média geral & & 6,0213
\end{tabular}

ns Não-significativo. * e**Significativo a $5 \%$ e $1 \%$ de probabilidade, respectivamente
Os valores médios do número de anéis de vasos laticíferos por clone variaram de 22,10 (IAC 302) a 46,00 (IAC 40) (Tabela 7). Os outros clones que apresentaram maior número de anéis de vasos laticíferos foram IAC 315, IAC 56 e IAC 306, com médias de $44,42,42,25$ e 41,00, respectivamente. Estudos conduzidos por Ho et al. (1973b) e Narayanan et al. (1974) provaram que o número de anéis de vasos laticíferos é o fator mais importante relacionado com a produção. Esse caráter, combinado com o índice de obstrução e perímetro, contribui com $75 \%$ da variação da produção em plantas jovens. Entretanto, segundo Premakumari \& Panikkar (1992), essa contribuição foi reduzida a $40 \%$ na fase adulta, a qual indicou um papel predominante da interação genótipo-ambiente.

Quanto à resistência ao cancro do painel de Colletotrichum, os clones IAC 40, IAC 302 , IAC 303, IAC 306, IAC 308, IAC 309, IAC 310 , IAC 311, IAC 312, IAC 313, IAC 314, IAC 315, IAC 316, IAC 317 e IAC 318 apresentaram alta resistência; e os clones IAC 56, IAC 300, IAC 301, IAC 307 e RRIM 600, resistência moderada.

Por meio do acompanhamento do hábito da troca de folhas, no ano de 1999 verificou-se que a troca de folhas se iniciou na segunda quinzena de julho e se encerrou no início de outubro. Os clones IAC 40, IAC 303, IAC 306, IAC 307, IAC 312, IAC 313, IAC 315, IAC 316, IAC 317 e RRIM 600 apresentaram o hábito regular de troca de folhas. Este hábito é um aspecto bastante importante em programas de seleção de clones para o controle de doenças foliares, como a antracnose e mal-das-folhas. Os clones IAC 56, IAC 302, IAC 309, IAC 310, IAC 311 , IAC 314 e IAC 318 apresentaram hábito irregular de troca de folhas, característica oriunda de híbridos de $H$. benthamiana. Os demais não trocaram de folhas dentro deste período e foram denominados de tardios (IAC 300, IAC 301 e IAC 308).

Em relação ao secamento do painel houve incidência de 7,0\% no clone IAC 302 e IAC 314, enquanto os clones IAC 40, IAC 308, IAC 309 apresentaram $6,0 \%$ de incidência em relação às árvores sangradas (Tabela 7). O secamento do painel é uma enfermidade fisiológica de seringueiras em regime de sangria, e que aumenta em alguns clones com intensidade de explotação (Bealing \& Chua, 1972). É 
Tabela 5. Dados médios de perímetro do caule referentes a primeira seleção de clones da série IAC, do experimento instalado no Núcleo de Agronomia do Noroeste do Instituto Agronômico (IAC), Votuporanga, SP.

\begin{tabular}{|c|c|c|c|c|c|c|c|c|c|c|c|}
\hline \multirow[t]{2}{*}{ Clone } & \multirow{2}{*}{$\begin{array}{l}\mathrm{N}^{\mathrm{o}} \mathrm{de} \\
\text { árvores }\end{array}$} & \multicolumn{7}{|c|}{ Período de imaturidade ${ }^{(1)}$} & \multicolumn{3}{|c|}{ Período adulto $^{(2)}$} \\
\hline & & Ano $1^{(3)}$ & Ano $2^{(4)}$ & Ano 3 & Ano 4 & Ano 5 & Ano 6 & Ano $7^{(5)}$ & Ano 8 & Ano 9 & Ano 10 \\
\hline IAC 40 & 18 & 7,54 & 10,42 & 19,83 & 27,02 & 35,80 & 43,15 & 48,50 & 51,90 & 58,78 & 61,33 \\
\hline IAC 56 & 17 & 6,35 & 8,94 & 16,92 & 23,83 & 33,23 & 41,29 & 47,96 & 51,70 & 57,21 & 60,93 \\
\hline IAC 300 & 17 & 5,73 & 8,13 & 15,11 & 22,44 & 28,14 & 36,86 & 42,35 & 43,55 & 52,59 & 56,21 \\
\hline IAC 301 & 18 & 7,29 & 10,68 & 17,81 & 25,28 & 31,14 & 37,33 & 42,75 & 44,70 & 51,19 & 53,02 \\
\hline IAC 302 & 16 & 6,97 & 11,41 & 18,78 & 29,67 & 38,75 & 43,97 & 47,95 & 51,56 & 59,42 & 60,02 \\
\hline IAC 303 & 18 & 7,78 & 11,24 & 18,22 & 25,92 & 33,79 & 40,61 & 46,20 & 48,83 & 57,47 & 63,00 \\
\hline IAC 306 & 15 & 6,42 & 9,44 & 17,39 & 25,74 & 32,22 & 38,01 & 43,16 & 43,28 & 49,94 & 56,96 \\
\hline IAC 307 & 18 & 7,38 & 11,28 & 18,67 & 29,29 & 36,47 & 43,05 & 47,00 & 51,00 & 59,25 & 60,64 \\
\hline IAC 308 & 17 & 5,79 & 7,41 & 14,45 & 23,65 & 32,35 & 38,95 & 45,20 & 50,55 & 57,60 & 60,81 \\
\hline IAC 309 & 18 & 7,17 & 11,50 & 20,22 & 30,04 & 37,47 & 43,80 & 49,16 & 52,28 & 59,11 & 65,16 \\
\hline IAC 310 & 15 & 5,70 & 7,81 & 13,56 & 20,37 & 28,55 & 34,83 & 41,10 & 45,73 & 55,56 & 63,10 \\
\hline IAC 311 & 15 & 6,84 & 10,01 & 17,82 & 26,25 & 35,86 & 41,83 & 48,81 & 51,67 & 59,00 & 65,10 \\
\hline IAC 312 & 14 & 8,15 & 13,20 & 22,80 & 28,47 & 35,40 & 42,08 & 50,50 & 54,54 & 62,85 & 64,00 \\
\hline IAC 313 & 17 & 7,30 & 10,17 & 17,45 & 25,55 & 31,33 & 39,67 & 44,22 & 48,17 & 55,86 & 59,32 \\
\hline IAC 314 & 16 & 6,25 & 9,54 & 17,13 & 26,24 & 33,96 & 40,21 & 45,76 & 50,61 & 58,44 & 60,36 \\
\hline IAC 315 & 18 & 7,09 & 9,93 & 18,45 & 21,07 & 37,70 & 43,96 & 50,90 & 53,85 & 61,95 & 65,10 \\
\hline IAC 316 & 14 & 7,32 & 11,02 & 19,00 & 26,68 & 33,69 & 37,25 & 45,51 & 47,72 & 53,61 & 57,39 \\
\hline IAC 317 & 15 & 6,42 & 9,43 & 15,56 & 17,14 & 26,00 & 31,50 & 37,81 & 41,25 & 47,50 & 50,00 \\
\hline IAC 318 & 17 & 6,29 & 6,70 & 12,15 & 20,93 & 29,45 & 36,40 & 42,25 & 46,00 & 52,70 & 62,07 \\
\hline RRIM $600^{(6)}$ & 60 & 5,39 & 9,42 & 10,64 & 17,53 & 26,61 & 33,34 & 39,28 & 44,24 & 51,06 & 54,10 \\
\hline Média geral & & $6,76 \pm 0,76$ & $9,88 \pm 1,58$ & $17,10 \pm 2,86$ & $24,66 \pm 3,75$ & $32,90 \pm 3,73$ & $39,40 \pm 3,65$ & $45,32 \pm 3,66$ & $48,66 \pm 3,88$ & $56,05 \pm 4,16$ & $59,93 \pm 4,20$ \\
\hline
\end{tabular}


Tabela 6. Incremento médio anual do crescimento do caule, em centímetros, referente a primeira seleção de clones da série IAC, do experimento instalado no Núcleo de Agronomia do Noroeste do Instituto Agronômico (IAC), Votuporanga, SP.

\begin{tabular}{|c|c|c|c|c|c|c|c|c|c|c|c|c|c|}
\hline \multirow[t]{2}{*}{ Clone } & \multirow{2}{*}{$\begin{array}{l}\mathrm{N}^{\mathrm{o}} \mathrm{de} \\
\text { árvores }\end{array}$} & \multicolumn{7}{|c|}{ Período de imaturidade ${ }^{(1)}$} & \multirow[t]{2}{*}{ Média } & \multicolumn{3}{|c|}{ Período adulto $^{(2)}$} & \multirow[t]{2}{*}{ Média } \\
\hline & & $0-1$ & $1-2$ & $2-3$ & $3-4$ & $4-5$ & $5-6$ & $6-7$ & & $7-8$ & $8-9$ & $9-10$ & \\
\hline IAC 40 & 18 & 7,54 & 2,88 & 9,38 & 9,52 & 8,78 & 7,35 & 5,35 & 7,26 & 3,40 & 6,88 & 2,55 & 4,28 \\
\hline IAC 56 & 17 & 6,35 & 2,59 & 7,98 & 9,44 & 9,40 & 8,06 & 6,67 & 7,21 & 3,74 & 5,51 & 3,72 & 4,32 \\
\hline IAC 300 & 17 & 5,73 & 2,40 & 6,98 & 9,26 & 5,70 & 8,72 & 5,49 & 6,33 & 1,20 & 9,04 & 3,62 & 4,62 \\
\hline IAC 301 & 18 & 7,29 & 3,39 & 7,13 & 7,45 & 5,86 & 6,19 & 5,42 & 6,10 & 1,95 & 6,49 & 1,83 & 3,42 \\
\hline IAC 302 & 16 & 6,97 & 4,44 & 7,37 & 10,89 & 9,08 & 5,22 & 3,98 & 6,85 & 3,61 & 7,86 & 0,60 & 4,02 \\
\hline IAC 303 & 18 & 7,78 & 3,46 & 6,98 & 7,70 & 7,87 & 6,82 & 5,59 & 6,60 & 2,63 & 8,64 & 5,53 & 5,60 \\
\hline IAC 306 & 15 & 6,42 & 3,90 & 7,95 & 8,35 & 6,48 & 5,79 & 5,15 & 6,29 & 0,12 & 6,66 & 7,02 & 4,60 \\
\hline IAC 307 & 18 & 7,38 & 4,03 & 7,39 & 9,46 & 7,18 & 6,58 & 3,95 & 6,57 & 4,00 & 8,25 & 1,39 & 4,55 \\
\hline IAC 308 & 17 & 5,79 & 1,62 & 7,04 & 9,20 & 8,70 & 6,60 & 6,25 & 6,46 & 5,35 & 7,05 & 3,21 & 5,20 \\
\hline IAC 309 & 18 & 7,17 & 4,33 & 8,72 & 7,82 & 7,43 & 6,33 & 5,36 & 6,74 & 3,12 & 6,83 & 6,05 & 5,33 \\
\hline IAC 310 & 15 & 5,70 & 2,11 & 5,75 & 6,08 & 8,18 & 6,28 & 6,27 & 5,77 & 4,63 & 9,83 & 7,54 & 7,33 \\
\hline IAC 311 & 15 & 6,84 & 3,17 & 7,81 & 8,64 & 9,61 & 5,97 & 6,98 & 7,00 & 2,86 & 7,33 & 6,10 & 5,43 \\
\hline IAC 312 & 14 & 8,15 & 5,05 & 9,60 & 6,03 & 6,93 & 6,68 & 8,42 & 7,27 & 4,04 & 8,31 & 1,15 & 4,50 \\
\hline IAC 313 & 17 & 7,30 & 2,87 & 7,28 & 8,10 & 5,78 & 8,34 & 4,55 & 6,32 & 3,95 & 7,69 & 3,46 & 5,03 \\
\hline IAC 314 & 16 & 6,25 & 3,29 & 7,59 & 7,18 & 7,72 & 6,25 & 5,55 & 6,26 & 4,85 & 7,83 & 1,92 & 4,87 \\
\hline IAC 315 & 18 & 7,09 & 2,84 & 8,52 & 2,62 & 16,63 & 6,26 & 6,94 & 7,27 & 2,95 & 8,10 & 3,15 & 4,73 \\
\hline IAC 316 & 14 & 7,32 & 3,70 & 7,98 & 7,68 & 7,01 & 3,56 & 8,26 & 6,50 & 2,21 & 5,89 & 3,78 & 3,96 \\
\hline IAC 317 & 15 & 6,42 & 3,01 & 6,13 & 1,58 & 8,86 & 5,50 & 6,31 & 5,40 & 3,44 & 6,25 & 2,50 & 4,06 \\
\hline IAC 318 & 17 & 6,29 & 0,41 & 5,45 & 8,78 & 8,52 & 6,95 & 5,85 & 6,04 & 3,75 & 6,70 & 9,37 & 6,61 \\
\hline RRIM $600^{(3)}$ & 60 & 5,39 & 4,03 & 1,22 & 6,89 & 9,08 & 6,73 & 5,94 & 5,61 & 4,96 & 6,82 & 3,04 & 4,94 \\
\hline Média geral & & $6,76 \pm 0,76$ & $3,18 \pm 1,06$ & $7,21 \pm 1,76$ & $7,63 \pm 2,25$ & $8,24 \pm 2,32$ & $, 51 \pm 1,13$ & $5,91 \pm 1,18$ & $6,49 \pm 0,55$ & $3,34 \pm 1,29$ & $7,40 \pm 1,09$ & $3,88 \pm 2,34$ & $4,87 \pm 0,91$ \\
\hline
\end{tabular}


possível que a presença de secamento do painel se deva ao aumento da concentração de estimulante no terceiro ano de sangria.

A quebra de árvores pelo vento é um caráter secundário de grande importância na avaliação do clone. Segundo Tan (1987), pode estar associada ao modelo de formação de copa do clone, altura da árvore, baixo índice de obstrução (fluxo prolongado do látex), propriedades da madeira. Quanto à incidência de quebra pelo vento, o clone IAC 306 classificou-se em primeiro lugar, com 14,0\% de quebra, seguido do IAC 314 e IAC 300 com 13,0\% e 12,0\% respectivamente, todos piores que a testemunha RRIM 600, que apresentou 5,0\% de quebra (Tabela 7).

Todos os clones selecionados apresentaram vigor, avaliado pelo perímetro do caule, espessura de casca virgem e número de anéis de vasos laticíferos superiores em relação ao clone testemunha RRIM 600, o que indica que o desempenho preliminar dos clones possibilita uma primeira seleção com vistas a testes em experimentos de avaliação de grande escala (Gonçalves et al., 1999), a serem instalados em várias regiões do Estado.

Os dados do primeiro ano de produção (Gonçalves et al., 1999) mostraram o desempenho dos clones do qual foi feita a primeira seleção com vistas a testá-los, em experimentos de avaliação de grande escala. Os clones IAC 40, IAC 56, IAC 300, IAC 301 e IAC 302 encontram-se devidamente instalados em várias regiões do Estado. A seleção, nesse caso, se baseou nas correlações existentes no primeiro ano de produção com as dos anos subseqüentes obtidas por Alika (1980), Ong \& Tan (1980) e Marques \& Gonçalves (1990).

O presente trabalho está além daquele publicado (Gonçalves et al., 1999) pela utilização de sistemas de sangria diferenciados, d/3 e d/4, com a aplicação do Ethrel no segundo e terceiro ano de sangria, respectivamente. É a primeira vez que essa prática de

Tabela 7. Dados médios de perímetro do caule, plantas aptas à sangria, espessura de casca e número de anéis de vasos laticíferos relativos a abertura do painel e porcentagem de árvores com seca de painel e quebradas pelo vento da primeira seleção de clones da serie IAC 300, realizada no Núcleo de Agronomia do Noroeste do Instituto Agronômico (IAC), Votuporanga, SP.

\begin{tabular}{|c|c|c|c|c|c|c|c|}
\hline \multirow[t]{2}{*}{ Clone } & \multirow{2}{*}{$\begin{array}{l}\mathrm{N}^{\mathrm{o}} \mathrm{de} \\
\text { árvores }\end{array}$} & \multicolumn{4}{|c|}{ Abertura do painel $^{(1)}$} & \multirow{2}{*}{$\begin{array}{c}\text { Árvores com } \\
\text { seca de } \\
\text { painel }(\%)\end{array}$} & \multirow{2}{*}{$\begin{array}{c}\text { Árvores } \\
\text { quebradas pelo } \\
\text { vento }(\%)\end{array}$} \\
\hline & & $\begin{array}{l}\text { Perímetro do } \\
\text { caule }(\mathrm{cm})\end{array}$ & $\begin{array}{l}\text { Plantas aptas } \\
\text { à sangria }(\%)\end{array}$ & $\begin{array}{l}\text { Espessura de } \\
\text { casca }(\mathrm{mm})\end{array}$ & $\begin{array}{c}\mathrm{N}^{\mathrm{o}} \text { de anéis de } \\
\text { vasos laticíferos }\end{array}$ & & \\
\hline IAC 40 & 18 & 48,50 & 78,00 & 5,75 & 46,00 & 6,00 & 0,00 \\
\hline IAC 56 & 17 & 47,96 & 76,00 & 7,21 & 42,25 & 0,00 & 6,00 \\
\hline IAC 300 & 17 & 42,35 & 60,00 & 6,00 & 30,20 & 0,00 & 12,00 \\
\hline IAC 301 & 18 & 42,75 & 61,00 & 6,20 & 30,72 & 0,00 & 0,00 \\
\hline IAC 302 & 16 & 47,95 & 40,00 & 5,70 & 22,10 & 7,00 & 0,00 \\
\hline IAC 303 & 18 & 46,20 & 95,00 & 6,20 & 30,90 & 0,00 & 6,00 \\
\hline IAC 306 & 15 & 43,16 & 87,00 & 6,81 & 41,00 & 0,00 & 14,00 \\
\hline IAC 307 & 18 & 47,00 & 83,00 & 6,22 & 32,10 & 0,00 & 0,00 \\
\hline IAC 308 & 17 & 45,20 & 88,00 & 6,30 & 34,01 & 6,00 & 0,00 \\
\hline IAC 309 & 18 & 49,16 & 89,00 & 6,50 & 23,80 & 6,00 & 0,00 \\
\hline IAC 310 & 15 & 41,10 & 80,00 & 6,81 & 36,30 & 0,00 & 0,00 \\
\hline IAC 311 & 15 & 48,81 & 87,00 & 6,99 & 27,65 & 0,00 & 0,00 \\
\hline IAC 312 & 14 & 50,50 & 92,00 & 6,60 & 30,60 & 0,00 & 0,00 \\
\hline IAC 313 & 17 & 44,22 & 76,00 & 6,18 & 34,08 & 0,00 & 0,00 \\
\hline IAC 314 & 16 & 45,76 & 81,00 & 6,45 & 32,55 & 7,00 & 13,00 \\
\hline IAC 315 & 18 & 50,90 & 100,00 & 7,50 & 44,42 & 0,00 & 0,00 \\
\hline IAC 316 & 14 & 45,51 & 93,00 & 6,00 & 29,00 & 0,00 & 0,00 \\
\hline IAC 317 & 15 & 37,81 & 20,00 & - & - & 0,00 & 0,00 \\
\hline IAC 318 & 17 & 42,25 & 76,00 & - & - & 0,00 & 0,00 \\
\hline RRIM $600^{(2)}$ & 60 & 39,28 & 83,00 & 6,23 & 29,06 & 3,00 & 5,00 \\
\hline Média geral & & $45,32+3,66$ & $77,25+19,28$ & $6,43+0,49$ & $33,15+6,64$ & $1,75+2,84$ & $2,80+4,86$ \\
\hline
\end{tabular}

${ }^{(1)} \mathrm{A}$ abertura do painel foi realizada no sétimo ano de desenvolvimento vegetativo. ${ }^{(2)}$ Testemunha. 
sangria é utilizada e teve como objetivo reduzir o ciclo de avaliação e seleção para uma melhor recomendação dos clones para plantio em pequena escala.

\section{Conclusões}

1. Os clones IAC 40, IAC 301, IAC 300, IAC 302 e IAC 56 apresentam melhor produção nos três primeiros anos de sangria do que o clone testemunha RRIM 600.

2. Quanto ao desenvolvimento do caule, na abertura do painel, os clones IAC 315 e IAC 312 apresentam o melhor desempenho.

3. Os clones IAC 40, IAC 302, IAC 303, IAC 306, IAC 308, IAC 309, IAC 310, IAC 311, IAC 312, IAC 313, IAC 314, IAC 315, IAC 316, IAC 317 e IAC 318 mostram alta resistência à antracnose-dopainel.

4. Os clones IAC 302 e 314 apresentam maior propensão ao secamento de painel de sangria.

5. Os clones IAC 306, IAC 314 e IAC 300 mostram maior incidência de quebra de árvores pelo vento.

\section{Agradecimentos}

Às laboratoristas, Andréa Cardoso Guerreiro e Lígia Regina Lima Gouvêa, pelas análises do sistema laticífero contido na casca da seringueira; aos técnicos agrícolas Ari de Camargo e Isaac de Souza, do Núcleo Experimental de Campinas e Estação Experimental de Votuporanga, respectivamente, pelo manejo aplicado ao experimento durante a fase de desenvolvimento do experimento.

\section{Referências}

ABRAHAM, P. D.; P'NG, T. C.; LEE, C. K. SIVAKUMARAN, S.; MANIKAM, B.; YEAH, C. P Ethrel stimulation of Hevea. In: INTERNATIONAL RUBBER CONFERENCE, 1975, Kuala Lumpur. Proceedings... Kuala Lumpur : Rubber Research Institute of Malaya, 1976. p. 347-350

ALIKA, J. E. Possibilities of early selection in Hevea brasiliensis. Silvae Genetica, Frankfurt, v. 29, n. 3/4 p. $161-162,1980$
BAJUNGU, H. E. Avaliação de perdas de colheitas causadas pelos patógenos. Piracicaba : ESALQ, 1977. $37 \mathrm{p}$

BEALING, F. J.; CHUA, S. E. Output, composition and metabolic activity of Hevea latex in relation to tapping intensity and the onset of brown blast. Journal of the Rubber Research Institute of Malaysia Journal, Kuala Lumpur, v. 23, n. 3, p. 204-231, 1972.

EMBRAPA. Centro Nacional de Pesquisa de Seringueira e Dendê (Manaus, AM). Melhoramento genético da seringueira. Manaus, 1989. 23 p. (Embrapa-CNPSD. Documentos, 10)

FURTADO, E. L. Comportamento decíduo da seringueira (Hevea spp.) e quantificação do mal-das-folhas, causado por Microcyclus ulei (P. Henn.) v. Arx. Piracicaba ESALQ, 1990. 82 p. Dissertação de Mestrado.

GONÇALVES, P. de S.; BORTOLETTO, N.; ORTOLANI, A. A.; BELLETI, G. O.; SANTOS W. R. dos. Desempenho de novos clones de seringueira. III. Seleções promissoras para a região de Votuporanga, Estado de São Paulo. Pesquisa Agropecuária Brasileira, Brasília, v. 34, n. 6, p. 971-980, jun. 1999

GONÇALVES, P. de S.; CARDOSO, M.; BORTOLETTO, N. Redução do ciclo de melhoramento e seleção na obtenção de cultivares de seringueira. Agronômico, Campinas, v. 40, n. 2 , p. $113-130,1988$

GONÇALVES, P. de S.; PAIVA, J. R. de; SOUZA, R. A de. Retrospectiva e atualidade do melhoramento genético da seringueira (Hevea spp.) no Brasil e em países asiáticos. Manaus : Embrapa-CNPSD, 1983. 69 p. (Embrapa-CNPSD. Documentos, 2)

HO, C. Y.; LEONG, Y. S.; JEYATHEVAN, V. Responses of clones and seedlings to stimulation in large-scale variety trials. In: RUBBER RESEARCH INSTITUTE OF MALAYSIA PLANTERS' CONFERENCE, 1973, Kuala Lumpur. Proceedings... Kuala Lumpur, 1973a. p. 101-121.

HO, C. Y.; NARAYANAN, R.; CHEN, K. T. Clonal nursery studies in Hevea. I. Nursery yields and associated structural characteristics and their variations. Rubber Research Institute of Malaysia Journal, Kuala Lumpur, v. 23, n. 4 , p. $305-326,1973$ b.

INTERNATIONAL RUBBER STUDY GROUP (Wembley, Grã-Bretanha). Consumption of natural rubber. Rubber Statistical Bulletin, Wembley, v. 53, n. 9 , p. $17-18,1999$

JONGE, P. de. Stimulation in Hevea brasiliensis III. Further observations on the effects of yield stimulants. 
Rubber Research Institute of Malaysia Journal, Kuala Lumpur, v. 14, p. 383-405, 1955

LEVANDOWSKY, D. W. Clonal variations in reaction of Hevea brasiliensis to stimulation of latex yield and to bark regeneration. In: NATURAL RUBBER RESEARCH CONFERENCE, 1961, Kuala Lumpur. Proceedings.. Kuala Lumpur : Rubber Research Institute of Malaysia, 1961. p. 270-281

MARQUES, J. R. B.; GONÇALVES, P. de S. Teste precoce de produção na seleção de plantas de seringueira Pesquisa Agropecuária Brasileira, Brasília, v. 25, n. 7, p. 1065-1077, jul. 1990.

NARAYANAN, R.; HO, C. Y; CHEN, K. T. Clonal nursery studies in Hevea. III. Correlations between yield, structural characters, latex constituents and plugging index. Rubber Research Institute of Malaysia Journal, Kuala Lumpur, v. 24, n. 1, p. $1-14,1974$.

ONG, S. H.; TAN, A. M. Correlations between yield girth and bark thickness of RRIM clones trials. Rubber Research Institute of Malaysia Journal, Kuala Lumpur v. 29, n. 1, p. $1-14,1980$

ONG, S. H.; SULTAN, M. O.; KHOO, S. K.; TAN, H. Performance of clones in RRIM 800 series (first selection) and promotion plot trials. In: PLANTERS' CONFERENCE, 1981, Kuala Lumpur. Proceedings..
Kuala Lumpur : Rubber Research Institute of Malaya, 1981. p. $340-355$.

PREMAKUMARI, D.; PANIKKAR, A. O. N. Anatomy and ultracytology of latex vessels. In: SETHURAJ, M. R.; MATHEW, N. M. (Ed.). Natural rubber: biology, cultivation and technology. Amsterdam : Elsevier, 1992. p. 67-87. (Developments in Crop Science, 23).

SÃO PAULO (Estado). Secretaria de Agricultura e Abastecimento. Comissão Técnica de Seringueira. A cultura da seringueira para o Estado de São Paulo. Campinas : CATI, 1999. 90 p. (Manual, 72).

TAN, H. Strategies in rubber tree breeding. In: ABBOT, A. J.; ATKIN, R. R. (Ed.). Improving vegetatively propagated crops. London : Academic, 1987. p. 27-62.

VAZQUEZ CORTEZ, J. A evolução da cultura da seringueira no Estado de São Paulo, Brasil. In: CICLO DE PALESTRAS SOBRE A HEVEICULTURA PAULISTA, 1., 1998., Barretos. Anais... Barretos: Secretaria de Agricultura e Abastecimento/Associação Paulista de Produtores e Beneficiadores de Borracha, 1999. p. 61-69.

VIRGENS FILHO, A. de C.; MATOS, C. R.; REIS, E. L.; SCOMPARIN, C. H. J. Cultivo de seringueira para produção de borracha natural. Viçosa : Centro de Produções Técnicas, 1999. 64 p. 